\title{
Electrophysiological Identification of Tonic and Phasic Neurons in Sensory Dorsal Root Ganglion and Their Distinct Implications in Inflammatory Pain
}

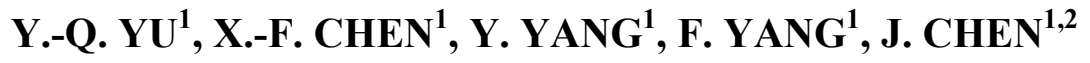 \\ ${ }^{1}$ Institute for Biomedical Sciences of Pain and Institute for Functional Brain Disorders, Tangdu \\ Hospital, Fourth Military Medical University, Xi'an, PR China, ${ }^{2}$ Key Laboratory of Brain Stress \\ and Behavior, PLA, Xi'an, PR China, ${ }^{3}$ Beijing Institute for Brain Disorders, Beijing, PR China
}

Received November 28, 2013

Accepted May 16, 2014

On-line August 26, 2014

\section{Summary}

In the mammalian autonomic nervous system, tonic and phasic neurons can be differentiated on firing patterns in response to long depolarizing current pulse. However, the similar firing patterns in the somatic primary sensory neurons and their functional significance are not well investigated. Here, we identified two types of neurons innervating somatic sensory in rat dorsal root ganglia (DRG). Tonic neurons fire action potentials (APs) in an intensity-dependent manner, whereas phasic neurons typically generate only one AP firing at the onset of stimulation regardless of intensity. Combining retrograde labeling of somatic DRG neurons with fluorescent tracer DiI, we further find that these neurons demonstrate distinct changes under inflammatory pain states induced by complete Freund's adjuvant (CFA) or bee venom toxin melittin. In tonic neurons, CFA and melittin treatments significantly decrease rheobase and AP durations (depolarization and repolarization), enhance amplitudes of overshoot and afterhyperpolarization (AHP), and increase the number of evoked action potentials. In phasic neurons, however, the same inflammation treatments cause fewer changes in these electrophysiological parameters except for the increased overshoot and decreased AP durations. In the present study, we find that tonic neurons are more hyperexcitable than phasic neurons after peripheral noxious inflammatory stimulation. The results indicate the distinct contributions of two types of DRG neurons in inflammatory pain.

\section{Key words}

Action potential • Dorsal root ganglion • Tonic neuron - Phasic neuron • Pain

\section{Corresponding author}

J. Chen, Institute for Biomedical Sciences of Pain and Institute for Functional Brain Disorders, Fourth Military Medical University, \#569 Xinsi Road, Baqiao, Xi'an 710038, PR China. Fax: +86 29 84777945. E-mail: junchen@fmmu.edu.cn

\section{Introduction}

Sensory information in the nervous system is mainly based on generation and propagation of a series of action potentials (APs) in neurons. In the adult mammalian nervous system, two major types of neurons can be differentiated on the basis of their distinct firing properties in response to long depolarizing current pulse: tonic neuron discharging APs throughout the current pulse and phasic neuron generally exhibiting only a single AP at the onset of stimulation. The similar firing patterns were widely reported in the autonomic nervous system, such as the parasympathetic ganglia (Myers et al. 1990), superior cervical ganglia (Jobling and Gibbins 1999, Malin and Nerbonne 2001, Jia et al. 2008), cardiac ganglia (Xi et al. 1994), thoracic ganglia (Jobling and Gibbins 1999), celiac ganglia (Wells and Mawe 1993, Dong et al. 2008), paracervical ganglia (Jobling and Lim 2008) and major pelvic ganglia (Kanjhan et al. 2003, Tan et al. 2003). These firing neurons in the autonomic nervous system act as a control system operating primarily below the level of consciousness and modulate sensory functions of visceral organs. Although the physiological significance 
of the different neuronal firing patterns was uncertain, previous studies demonstrated that tonic or phasic neurons could change neuronal excitabilities following pathological stimulation. It was reported that the increased excitability of phasic neuron contributed to cystitis-induced pain and hyperactivity of the urinary bladder (Yoshimura and de Groat 1999, Sculptoreanu and de Groat 2007). In contrast, Dong et al. (2008) found that intestinal inflammation induced by trinitrobenzene sulfonate selectively increased neuronal excitability of tonic, but not phasic, type of celiac ganglion neurons. Ditting et al. (2009) also suggested that the greater susceptibility of renal afferent neurons correlated with the higher rate of tonic firings and further contributed to inflammatory process in the kinedy. These results are of special interests since the electrophysiological properties of these neurons are likely to be state-dependent under pathological conditions and might involve in hyperreflexic conditions of visceral organs.

Although tonic and phasic neurons in rat dorsal root ganglia were previously reported (Yoshimura and de Groat 1999, Sculptoreanu and de Groat 2007), these neurons were isolated from L4-S3 DRGs containing both visceral and somatic components. It was shown that passive and active electrophysiological properties between visceral and somatic sensory neurons were remarkable distinct (Gold and Traub 2004). In the present study, we explore whether the similar firing patterns exist in the somatic primary sensory neurons and further investigate their potential contributions in inflammatory pain. Together with retrograde labeling method and patch-clamp recording in somatic dorsal root ganglion neurons of lumbar segment (L4-6), we report that tonic and phasic neurons not only differently change the neuronal excitabilities but also might exert distinct implications in somatic inflammatory pain.

\section{Materials and Methods}

\section{Experimental animals}

The experiments were performed on male Sprague-Dawley albino rats (purchased from Laboratory Animal Center of the Fourth Military Medical University) weighing $80-120 \mathrm{~g}$ (age >3 postnatal weeks). The animals had access to water and food $a d$ libitum, and were maintained at room temperature (22$26{ }^{\circ} \mathrm{C}$ ) with a light/dark cycle of $12 \mathrm{~h}$. The experimental procedures were approved by the Institutional Animal
Care and Use Committee of FMMU. The number of animals used and their sufferings were minimized. The experimental procedures were approved by the Institutional Animal Care and Use Committee of FMMU.

\section{Retrograde labeling of somatic DRG neurons}

DRG neurons (L4-6) innervating the glabrous skin of left hind paw of the rats were labeled with the retrograde tracer 1, 1'-dioctadecyl-3, 3, 3', 3'tetramethylindocarbo-cyanine perchlorate (DiI, Fluka, Japan). Labeling was performed as previously described (Yu et al. 2013). Briefly, DiI was dissolved in dimethyl sulfoxide $(170 \mathrm{mg} / \mathrm{ml})$ and then diluted to $17 \mathrm{mg} / \mathrm{ml}$ with $0.9 \%$ sterile saline. $20 \mu$ diluted dye was injected subcutaneously (s.c.) at three to five sites in glabrous skin with a $50 \mu$ l Hamilton syringe. DiI-labeled neurons were identified under fluorescence microscope (excitation, $550 \mathrm{~nm}$; emission, $650 \mathrm{~nm}$ ).

\section{Inflammatory pain models and behavioral test}

We established two inflammatory pain models two weeks after DiI labeling. Complete Freund's adjuvant (CFA, $100 \mu 1,1: 1$ dissolved in $0.9 \%$ sterile saline) was administrated into the plantar surface of rat left hindpaw to induce inflammatory pain one day after CFA injection ( $\mathrm{Yu}$ et al. 2011). Melittin $(50 \mu \mathrm{g} / 50 \mu \mathrm{l}$, dissolved in $0.9 \%$ sterile saline) was injected into the plantar surface of rat left hindpaw to induce acute inflammatory pain within two hours as previously reported (Yu et al. 2009).

All behavioral tests were performed by observers blinded to the experimental conditions as previously reported ( $\mathrm{Yu}$ et al. 2009). To assess heat hypersensitivity, rats were placed in a plastic chamber on the surface of a $2 \mathrm{~mm}$ thick glass plate and the sensitivity to heat stimuli was detected by RTY-3 radiant heat stimulator (Xi'an Bobang Technologies of Chemical Industry Co. Ltd., China). The heat stimuli were applied to both the injection site and the corresponding area of the contralateral paw, and the latency was determined as the duration from the beginning of heat stimuli to the occurrence of a marked withdrawal reflex. Five stimuli were repeated for each site and the latter three or four values were averaged as mean paw withdrawal thermal latency (PWTL, s). For evaluation of mechanical hypersensitivity, mechanical stimuli were applied by using ascending graded individual von Frey monofilaments with different 
bending forces. A bending force being able to evoke $50 \%$ occurrence of paw withdrawal reflex was expressed as the paw withdrawal mechanical threshold (PWMT, mN).

\section{Whole cell patch-clamp electrophysiology}

DRGs of the lumbar segment (L4-6) were harvested for study 1 day after CFA injection or $2 \mathrm{~h}$ after melittin treatment based on our recent studies ( $\mathrm{Yu}$ et al. 2011, 2013). DRGs were removed and chopped in half, incubated for $40 \mathrm{~min}$ at $37^{\circ} \mathrm{C}$ with DMEM solution (Sigma-Aldrich, Saint Louis, MO, USA) containing $1 \mathrm{mg} / \mathrm{ml}$ collagenase (type IA, Sigma) and $0.4 \mathrm{mg} / \mathrm{ml}$ trypsin (type I, Sigma). After three washes in standard external solution, individual cells were dispersed by trituration with a fire-polished Pasteur pipette and plated on glass cover slips. Experiments were carried out within $8 \mathrm{~h}$ and these cells retained a healthy appearance and had negative resting potentials and overshooting action potentials. All recordings were made with EPC10 amplifier and Pulse software (HEKA Elektronik, Germany). The data were analyzed by Igor software (Configuration Metrics, Inc. Oregon, USA). Patch electrodes fabricated with P-97 Puller (Narishige, Japan) had resistances of 3-5 M $\Omega$. After G $\Omega$-seal wholecell formed at room temperature $\left(20-22{ }^{\circ} \mathrm{C}\right)$ under voltage-clamp holding at $-70 \mathrm{mV}$, capacitance transient was cancelled, series resistance was compensated $(>80 \%)$ and leak current was subtracted digitally. For current clamp recording of action potentials (APs), the standard external solution contained (in $\mathrm{mM}$ ): $150 \mathrm{NaCl}, 5 \mathrm{KCl}, 1 \mathrm{MgCl}_{2}, 2.5 \mathrm{CaCl}_{2}, 10 \mathrm{HEPES}$ and 10 glucose (pH 7.4 with $\mathrm{NaOH}$ ) and the internal solution contained (in $\mathrm{mM}$ ): $150 \mathrm{KCl}, 1 \mathrm{MgCl}_{2}$, 10 HEPES and $4 \mathrm{Mg}$-ATP, adjusted to $\mathrm{pH} 7.4$ with $\mathrm{KOH}$ (310 mOsm).

To identify the firing patterns in response to different depolarizing currents, the first rectangular prepulse $(3 \mathrm{~ms})$ and the second pulse $(500 \mathrm{~ms})$ with interpulse at the holding potential were applied with different intensity (100 pA, 200 pA, 400 pA, 500 pA). To analyze the potential effects of inflammatory pain on electrophysiological properties of DiI-labeled DRG neurons, we measured the first prepulse ( $3 \mathrm{~ms}, 500 \mathrm{pA})$ evoked AP parameters including capacitance, resting membrane potential (RMP), rheobase (minimal current required to evoke an action potential), overshoot, depolarization duration, repolarization duration, afterhyperpolarization (AHP) amplitude. The number of APs was abtained by the depolarizing current pulse with $500 \mathrm{~ms}$ and $500 \mathrm{pA}$.

\section{Data analysis}

All data were expressed as means \pm SEM. Statistical comparisons were performed using one-way ANOVA followed by Fisher's PLSD test. Statistical significance was indicated by a $\mathrm{P}$ value $<0.05$.

\section{Results}

\section{Tonic and phasic types of DRG neurons}

In L4-6 DRG neurons of normal rats, we detected two types of firing patterns in response to long depolarizing currents (duration: $500 \mathrm{~ms}$; intensity: 100, 200, 400 and $500 \mathrm{pA}$ ). As shown in Figure 1A and B, tonic neurons discharged action potentials (APs) throughout the current pulse and the average number of APs were 3 spikes, 7 spikes and 8 spikes in response to stimulus intensities of $200 \mathrm{pA}, 400 \mathrm{pA}$ and $500 \mathrm{pA}$, respectively $(n=18)$. However, phasic neurons mainly discharged a single AP at the onset of the same series of stimuli ( $\mathrm{n}=17)$. The recorded DRG neurons of both tonic and phasic types were mainly in the size range between 20-30 $\mu \mathrm{m}$ in diameter (belong to small- or mediumsized neurons) and there was no significant difference in size distributions (Fig. 1C). Therefore, the size of DRG neuron generated nearly no effect on neuronal firing properties.

\section{Inflammatory pain models}

Then we established two models of inflammatory pain induced by CFA and melittin (Fig. 2). One day after intraplantar injection of CFA (100 $\mu \mathrm{l})$, paw withdrawal thermal latency (PWTL) reduced from $11.79 \pm 0.87$ to $7.81 \pm 1.00 \mathrm{~s}(\mathrm{n}=7, \mathrm{p}<0.05)$ and paw withdrawal mechanical threshold (PWMT) decreased from $28.57 \pm 1.80$ to $9.11 \pm 0.60 \mathrm{~g} \quad(\mathrm{n}=7$, $\mathrm{p}<0.001)$. Similarly, melittin treatment $(50 \mu \mathrm{g} / 50 \mu \mathrm{l})$ produced significant changes in PWTL (from $11.41 \pm 1.10$ to $6.93 \pm 1.11 \mathrm{~s}, \mathrm{n}=6, \mathrm{p}<0.05)$ and PWMT (from $28.0 \pm 3.74$ to $9.7 \pm 1.56 \mathrm{~g}, \mathrm{n}=6, \mathrm{p}<0.01$ ) after $2 \mathrm{~h}$ of injection. The results demonstrated the establishment of heat and mechanical hypersensitivity following CFA or melittin inflammation treatment. 

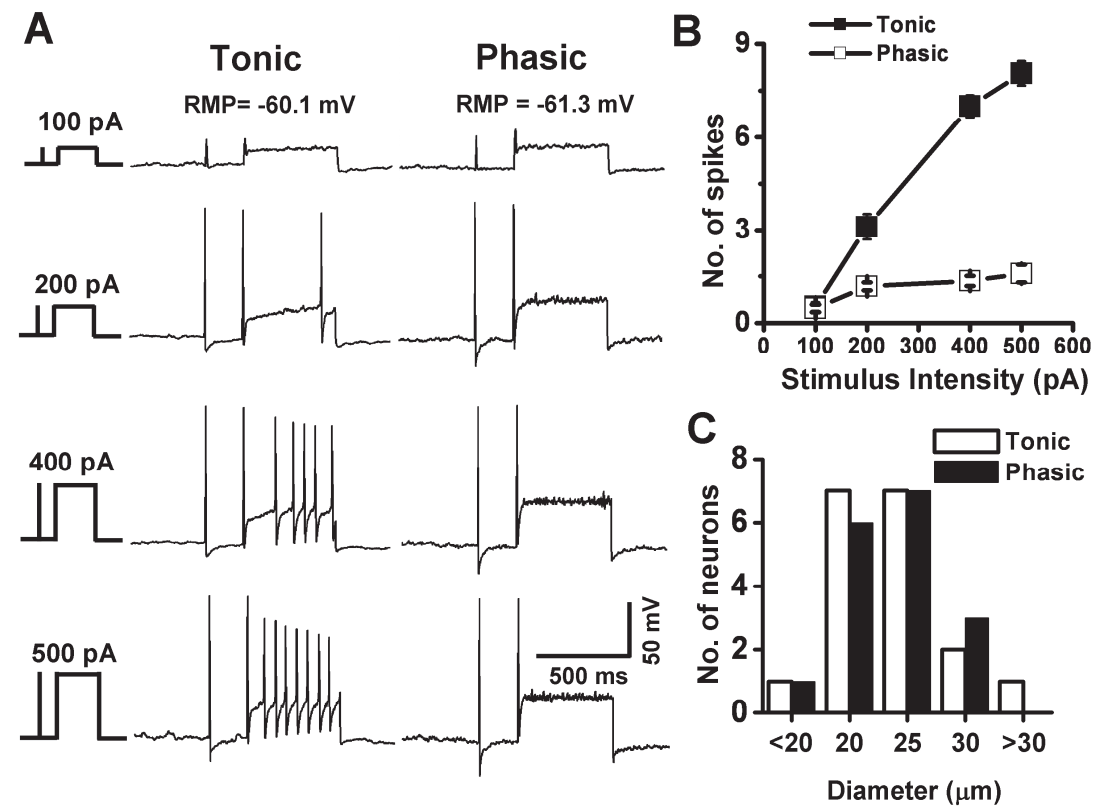

Fig. 1. Electrophysiological identification of tonic and phasic types of DRG neurons. (A) Example showed that different current injections (intensity, 100, 200, 400 and $500 \mathrm{pA}$; duration, $3 \mathrm{~ms}$ and $500 \mathrm{~ms}$ with a $200 \mathrm{~ms}$ interpulse at the holding potential, left panel) caused tonic firings (middle panel) or phasic firings (right panel) in DRG neurons of normal rats. RMP, resting membrane potential. (B) Stimulus intensity-response curves of tonic and phasic types of DRG neurons. Tonic neurons increased the number of AP firings in response to the graded suprathreshold stimuli. In contrast, phasic neurons had nearly no changes in AP firings. (C) Both tonic and phasic types of DRG neurons were mainly between 20-30 $\mu \mathrm{m}$ in diameter and there was no significant difference in size distributions between two types of neurons.

\section{Retrograde labeling of DRG neurons}

To analyze the potential effects of inflammatory treatments (CFA and melittin) on excitabilities of tonic and phasic types of DRG neurons, we used retrograde tracer 1, 1'-dioctadecyl-3, 3, 3', 3'-tetramethylindocarbocyanine perchlorate (DiI) to identify the cell bodies of DRG neurons innervating the glabrous skin of rat hindpaw as previously described (Yu et al. 2013). Two weeks after DiI injection, distribution of the tracer in dissociated DRG neurons could be identified under fluorescence illumination (Fig. 3). Our unpublished data showed DiI labeled DRG neurons could not co-express parvalbumin, a highly specific molecular marker for proprioceptors in large DRG (Ichikawa et al. 1994, Ji et al. 2012), indicating the retrograde labelling methods introduced in the present study could not label primary proprioceptors in DRG through muscle spindle afferent in rat hindpaw.
A

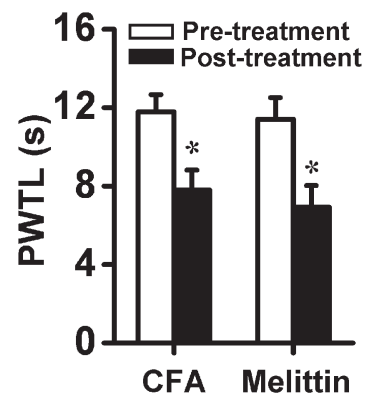

B

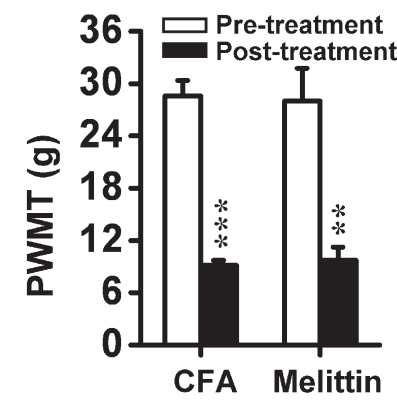

Fig. 2. Inflammatory pain models induced by complete Freund's adjuvant and melittin. PWTL (A) and PWMT (B) were significantly reduced following inflammation treatment by complete Freund's adjuvant $(100 \mu \mathrm{l})$ or melittin $(50 \mu \mathrm{g} / 50 \mu \mathrm{l})$, indicating the establishment of heat and mechanical pain hypersensitivity. PWTL, paw withdrawal thermal latency. PWMT, paw withdrawal mechanical threshold. $*, \mathrm{p}<0.05$; **, $\mathrm{p}<0.01$; $* * *, \mathrm{p}<0.001$.
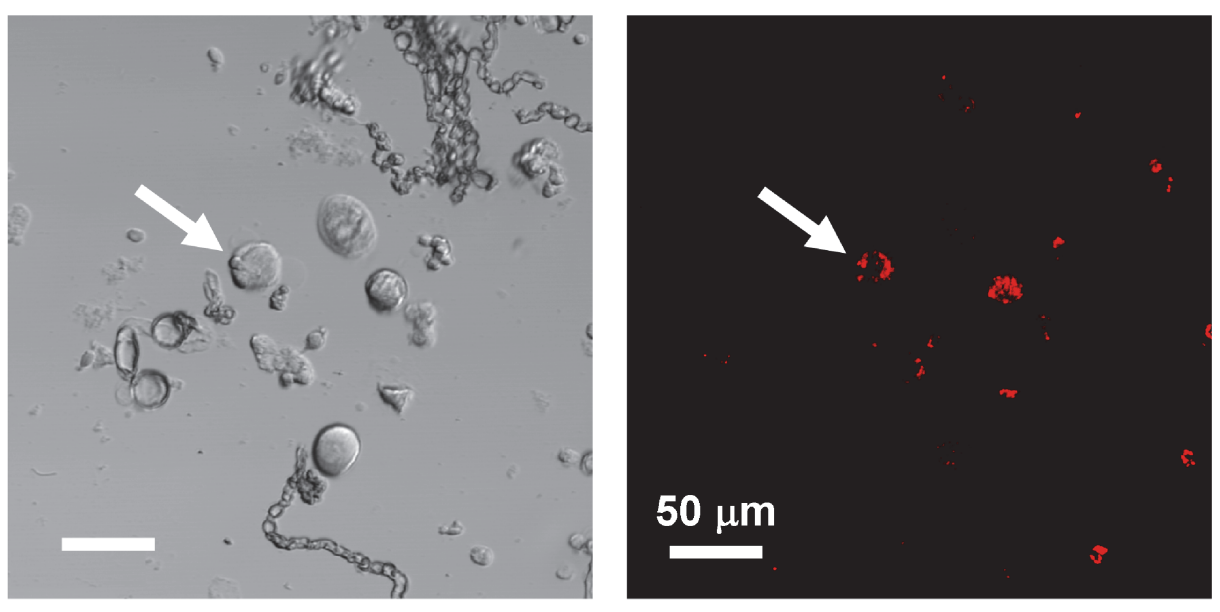

Fig. 3. Retrograde fluorescent labeling of DRG neurons innervating the glabrous skin of rat hindpaw. Two weeks after s.c. injection of $20 \mu \mathrm{l}$ DiI $(17 \mathrm{mg} / \mathrm{ml}$, dissolved in $10 \%$ DMSO), DiIlabeled neurons acutely dissociated from L4-6 DRGs were demonstrated under phasecontrast (A) and fluorescent photomicrograph (B). Arrows indicated the DRG neuron for patch-clamp recording. 
Effects of inflammatory pain on tonic and phasic DRG neurons

We further examined the effects of inflammatory pain on electrophysiological properties of tonic and phasic DRG neurons labeling with DiI (Table 1). Tonic and phasic neurons showed different changes after CFA or melittin treatment. In tonic neurons, CFA and melittin inflammatory treatments significantly decreased rheobase, depolarization and repolarization durations, enhanced amplitudes of overshoot and afterhyperpolarization (AHP) and increased the number of AP firings. In phasic neurons, the same inflammation treatments caused changes only in overshoot and AP durations, but not in rheobase, AHP or the number of AP firing. Neither capacitance (Cap) nor resting membrane potential (RMP) in two types of DRG neurons was affected by inflammatory pain.

Table 1. Effects of inflammation treatment on electrophysiological properties of DiI-labeled DRG neurons.

\begin{tabular}{|c|c|c|c|c|c|c|c|c|}
\hline Neural type & $\begin{array}{l}\text { Cap } \\
(p F)\end{array}$ & $\begin{array}{l}\text { RMP } \\
(\mathrm{mV})\end{array}$ & $\begin{array}{c}\text { Rheobase } \\
\text { (pA) }\end{array}$ & $\begin{array}{l}\text { Overshoot } \\
(\mathrm{mV})\end{array}$ & $\begin{array}{c}\text { Dep } \\
\text { duration } \\
(\mathrm{ms})\end{array}$ & $\begin{array}{c}\text { Rep } \\
\text { duration } \\
(\mathrm{ms})\end{array}$ & $\begin{array}{l}\text { AHP } \\
(\mathrm{mV})\end{array}$ & $\begin{array}{c}\text { No. of APs } \\
\text { at } 500 \text { pA }\end{array}$ \\
\hline \multicolumn{9}{|l|}{ Tonic } \\
\hline Control $(n=13)$ & $22.2 \pm 1.8$ & $-60.3 \pm 0.7$ & $128.6 \pm 6.9$ & $80.0 \pm 2.3$ & $1.5 \pm 0.0$ & $2.6 \pm 0.1$ & $-25.7 \pm 0.7$ & $7.0 \pm 1.0$ \\
\hline$C F A(n=15)$ & $24.4 \pm 1.3$ & $-58.5 \pm 0.7$ & $103.6 \pm 3.6^{* *}$ & $91.5 \pm 4.0 * *$ & $1.3 \pm 0.0 * * *$ & $1.9 \pm 0.1 * *$ & $-40.4 \pm 3.6^{* *}$ & $11.9 \pm 0.9 *$ \\
\hline Melittin $(n=17)$ & $21.1 \pm 1.0$ & $-58.1 \pm 1.0$ & $102.6 \pm 6.0 * *$ & $89.9 \pm 2.7^{* *}$ & $1.3 \pm 0.0 * * *$ & $2.0 \pm 0.1 * *$ & $-35.2 \pm 2.9^{*}$ & $12.2 \pm 1.4^{*}$ \\
\hline \multicolumn{9}{|l|}{ Phasic } \\
\hline Control $(n=13)$ & $21.5 \pm 1.5$ & $-60.5 \pm 1.1$ & $145.8 \pm 7.4$ & $75.5 \pm 4.0$ & $1.6 \pm 0.0$ & $2.2 \pm 0.15$ & $-33.3 \pm 3.3$ & $1.5 \pm 0.3$ \\
\hline$C F A(n=12)$ & $25.5 \pm 1.7$ & $-61.5 \pm 0.9$ & $143.8 \pm 8.9$ & $88.8 \pm 4.7 * *$ & $1.3 \pm 0.0 * *$ & $1.7 \pm 0.1 * *$ & $-40.7 \pm 5.7$ & $1.3 \pm 0.2$ \\
\hline Melittin $(n=18)$ & $24.3 \pm 1.9$ & $-60.8 \pm 0.6$ & $154.2 \pm 4.2$ & $87.2 \pm 2.5^{* *}$ & $1.5 \pm 0.1$ & $1.8 \pm 0.1 *$ & $-35.0 \pm 3.7$ & $1.4 \pm 0.3$ \\
\hline
\end{tabular}

Cap, capacitance; RMP, resting membrane potential; Dep, depolarization; Rep, repolarization; AHP, afterhyperpolarization; AP, action potential. ${ }^{*} \mathrm{P}<0.05, * * \mathrm{P}<0.01, * * * \mathrm{P}<0.001$ vs. control. Numerical in parenthesis indicates the number of neurons.

\section{Discussion}

We got following results in the present study. First, two types of DRG neurons were identified in somatic primary sensory neurons. Tonic neuron exerted constant action potential firings in an intensity-dependent manner, whereas phasic neuron typically generated only one AP at the onset of stimulation regardless of intensity. Second, tonic and phasic neurons exhibited quite distinguishable changes in electrophysiological properties under inflammatory pain states. In tonic neurons, CFA and melittin inflammation treatments significantly decreased rheobase and AP durations (depolarization and repolarization), enhanced amplitudes of overshoot and afterhyperpolarization (AHP) and increased the number of action potential firings. In phasic neurons, however, the same treatments caused less change in these electrophysiological parameters except for increased overshoot and decreased AP durations. We concluded that tonic neurons and phasic neurons not only could change their neuronal excitabilities but might perform distinct implications in inflammatory pain.

At present, most investigations support that sensory information in nervous system is dependent on action potentials that propagate along neuronal axons (Debanne 2004) and transmit through synapses (Kampa 2007). Little concern is paid on the regulation of sensory information mediated by action potentials in soma itself. Results from spinal cord indicated that different neuronal firing patterns performed distinct nociceptive sensory processing with unknown mechanism (Ruscheweyh and Sandkuhler 2002, Prescott and De Koninck 2002). Previous investigations showed that the width and amplitude of presynaptic action potential were of fundamental importance in determining the strength of synapses and the response of short-term synaptic depression (Debanne 2004). Here, we further showed that peripheral inflammatory pain caused significant changes in the electrophysiological properties (such as AP amplitude and repolarization durations) of somatic DRG neurons. The results imply that action potential firings in primary sensory neurons are not only state-dependent, but 
also significant for the modulation of pathological pain.

Except for remarkable difference in neuronal firing abilities, tonic neurons had lower current threshold $(17 \mathrm{pA})$ and AHP amplitude $(7 \mathrm{mV})$ than phasic neurons. Our data also suggested that tonic neurons seemed to be more hyperexcitable than phasic neurons following inflammation treatments. After CFA and melittin treatments, tonic neurons decreased rheobase from $129 \mathrm{pA}$ to $104 \mathrm{pA}$ and $103 \mathrm{pA}$, increased the number of AP firings from 7 spikes to 12 spikes and 12 spikes. In contrast, the same inflammation treatments had negligible effects on rheobase and AP firings in phasic neurons. The inherent difference between tonic and phasic neurons in both physical and pathological conditions indicated that neuron subtype-based therapy might be a potential strategy against pathological pain.

The other concern rising from present study is whether inflammation treatment can change neuronal phenotypes between phasic and tonic neurons. Our data showed that the percentage of tonic and phasic neurons is $50 \%$ (13 out of 26$)$ and $50 \%$ (13 out of 26 ) in the control group. After CFA or melittin treatment, the percentage of tonic neuron was $56 \%$ (15 out of 27) or $49 \%$ (17 out of 35$)$ and the percentage of phasic neuron was $44 \%$ (12 out of 27$)$ or $51 \%$ (18 out of 35$)$. The results indicated that the proportions of tonic and phasic neurons were not significantly changed by CFA and melittin inflammation. Sculptoreanu and de Groat (2007) reported that substance $\mathrm{P}$ and neurokinin $\mathrm{A}$ increased peak firings of phasic neurons (from 1 to 5 spikes) and of tonic neurons (from 12 to 15 ). It should be noted that increased firings of phasic neuron were not half of that in naive tonic neurons (Yoshimura and de Groat 1999, Sculptoreanu and de Groat 2007). Additionally, the chemical substrates of phasic and tonic neuron were quite different. The former was neurofilament negative and tetrodotoxin-resistant sodium channel positive, whereas expressions of these markers were just contrast in the latter (Yoshimura and de Groat 1999, Sculptoreanu and de Groat 2007). These results indicate that the possibility of inflammation-induced switch from phasic to tonic neuron, if exist, is quite limited.

In summary, in primary somatic sensory system, we firstly identify tonic and phasic neurons that exert distinct implications following inflammatory pain. At present, an important unanswered question is the molecule(s) determining different neuronal firing properties. Nevertheless, the molecule(s) dominating neuron subtypes might be the preferred targets for pathological pain control.

\section{Conflict of Interest}

There is no conflict of interest.

\section{Acknowledgements}

This work was supported by grants from National Basic Research Program of China (2013CB835100, 2011CB504100), National Key Technology R\&D Program (2013BAI04B04), the NSFC (31100803, 81102064, 81171049), the Twelfth Five-Year project (AWS12J004) and Science and Technology Program of Yunnan Province (2013GA003).

\section{References}

DEBANNE D: Information processing in the axon. Nat Rev Neurosci 5: 304-316, 2004.

DITTING T, TIEGS G, RODIONOVA K, REEH PW, NEUHUBER W, FREISINGER W, VEELKEN R: Do distinct populations of dorsal root ganglion neurons account for the sensory peptidergic innervation of the kidney? $\mathrm{Am}$ J Physiol Renal Physiol 297: F1427-F1434, 2009.

DONG XX, THACKER M, PONTELL L, FURNESS JB, NURGALI K: Effects of intestinal inflammation on specific subgroups of guinea-pig celiac ganglion neurons. Neurosci Lett 444: 231-235, 2008.

GOLD MS, TRAUB RJ: Cutaneous and colonic rat DRG neurons differ with respect to both baseline and PGE2-induced changes in passive and active electrophysiological properties. $J$ Neurophysiol 91: 2524-2531, 2004.

ICHIKAWA H, DEGUCHI T, NAKAGO T, JACOBOWITZ DM, SUGIMOTO T: Parvalbumin, calretinin and carbonic anhydrase in the trigeminal and spinal primary neurons of the rat. Brain Res 655: 241-245, 1994.

JI ZG, ITO S, HONJOH T, OHTA H, ISHIZUKA T, FUKAZAWA Y, YAWO H: Light-evoked somatosensory perception of transgenic rats that express channelrhodopsin-2 in dorsal root ganglion cells. PLoS One 7: e32699, 2012. 
JIA Z, BEI J, RODAT-DESPOIX L, LIU B, JIA Q, DELMAS P, ZHANG H: NGF inhibits M/KCNQ currents and selectively alters neuronal excitability in subsets of sympathetic neurons depending on their M/KCNQ current background. J Gen Physiol 131: 575-587, 2008.

JOBLING P, GIBBINS IL: Electrophysiological and morphological diversity of mouse sympathetic neurons. J Neurophysiol 82: 2747-2764, 1999.

JOBLING P, LIM R: Anatomical and physiological properties of pelvic ganglion neurons in female mice. Auton Neurosci 140: 30-39, 2008.

KAMPA BM, LETZKUS JJ, STUART GJ: Dendritic mechanisms controlling spike-timing-dependent synaptic plasticity. Trends Neurosci 30: 456-463, 2007.

KANJHAN R, OSBORNE PB, OUYANG M, KEAST JR: Postnatal maturational changes in rat pelvic autonomic ganglion cells: a mixture of steroid-dependent and -independent effects. J Neurophysiol 89: 315-323, 2003.

MALIN SA, NERBONNE JM: Molecular heterogeneity of the voltage-gated fast transient outward $\mathrm{K}^{+}$current, I(Af), in mammalian neurons. J Neurosci 21: 8004-8014, 2001.

MYERS AC, UNDEM BJ, WEINREICH D: Electrophysiological properties of neurons in guinea pig bronchial parasympathetic ganglia. Am J Physiol 259: L403-L409, 1990.

PRESCOTT SA, DE KONINCK Y: Four cell types with distinctive membrane properties and morphologies in lamina I of the spinal dorsal horn of the adult rat. $J$ Physiol 539: 817-836, 2002.

RUSCHEWEYH R, SANDKUHLER J: Lamina-specific membrane and discharge properties of rat spinal dorsal horn neurones in vitro. $J$ Physiol 541: 231-244, 2002.

SCULPTOREANU A, DE GROAT WC: Neurokinins enhance excitability in capsaicin-responsive DRG neurons. Exp Neurol 205: 92-100, 2007.

TAN H, MAWE GM, VIZZARD MA: Electrical properties of neurons in the intact rat major pelvic ganglion. Auton Neurosci 134: 26-37, 2007.

WELLS DG, MAWE GM: Physiological and morphological properties of neurons in sphincter of Oddi region of the guinea pig. Am J Physiol 265: G258-G269, 1993.

XI X, RANDALL WC, WURSTER RD: Electrophysiological properties of canine cardiac ganglion cell types. J Auton Nerv Syst 47: 69-74, 1994.

YOSHIMURA N, DE GROAT WC: Increased excitability of afferent neurons innervating rat urinary bladder after chronic bladder inflammation. $J$ Neurosci 19: 4644-4653, 1999.

YU YQ, ZHAO F, CHEN J: Activation of ERK1/2 in the primary injury site is required to maintain melittin-enhanced wind-up of rat spinal wide-dynamic-range neurons. Neurosci Lett 459: 137-141, 2009.

YU YQ, ZHAO F, GUAN SM, CHEN J: Antisense-mediated knockdown of Na(V)1.8, but not Na(V)1.9, generates inhibitory effects on complete Freund's adjuvant-induced inflammatory pain in rat. PLoS One 6: e19865, 2011.

YU YQ, ZHAO ZY, CHEN XF, XIE F, YANG Y, CHEN J: Activation of tetrodotoxin-resistant sodium channel NaV1.9 in rat primary sensory neurons contributes to melittin-induced pain behavior. Neuromolecular Med $\mathbf{1 5}$ : 209-217, 2013. 